Benedikt Vogel*

\title{
Die geheimen Lehrschriften zur Duft-Kunst (Kōdō hidensho) - Ästhetik und Praxis der Duft-Kunst in der Edo-Zeit
}

https://doi.org/10.1515/asia-2019-0009

\section{The Secret Teachings on the Art of Incense (Kōdō hidensho) - Aesthetics and Practice of the Art of Incense in the Edo-period}

\begin{abstract}
The Incense Ceremony is one of Japans traditional arts. After the early court enjoyed fragrance and incense in a playful manner (as documented by sources dating back as far as the early eighth century), it evolved during the fifteenth century into a complex ceremony. The moment of olfactory perception turned into a strict ritual, which combined performative as well as ornamental constituents. In the following Edo period (1603-1868) the Incense Ceremony is characterized by a significant rise in popularity, marked by a growing number of practitioners, who used written treatises to teach and transmit practical knowledge about the art. As one of the first printed treatises the Kōdo hidensho 香道秘伝書 (1669) reached wide circulation. Additionally, the number of comments on this specific treatise published in the century following its publication attest to its high status. While there are other treatises focusing on some aspects of the ceremony in more detail, the Kōdo hidensho does provide a solid overview on the ceremony, its material and performative constituents, its games and how they were celebrated. Consisting of nine separate parts in its entirety, the following translation presents the first two.
\end{abstract}

Keywords: incense, aesthetics, kōdō, performance, Edo period

Schlagworte: Räucherwerk, Ästhetik, kōdō, Aufführungspraxis, Edo-Zeit

Der Umgang mit Düften kann in Japan auf eine lange Geschichte zurückblicken. Datiert das Nihon shoki (710) den wohl eher legendenhaften ersten Kontakt Japans mit Duftholz auf das Jahr 595, ${ }^{1}$ illustrieren bereits im frühen 8 .

1 Vgl. Aston (Übers.) 1997: 123.

*Corresponding author: Benedikt Vogel, Trier University, Trier, Germany.

E-mail:vogel@uni-trier.de 
Jahrhundert Rezeptbücher ${ }^{2}$ und Handelsregister ${ }^{3}$ den Umgang der adeligen Gesellschaft mit angemischtem Räucherwerk (takimono/nerikō). Das Spiel mit eigenen Duftkreationen stellte am Hofe eine beliebte Beschäftigung dar und das Räuchern von Gewändern, Haaren und Briefen spiegelte die individuelle ästhetische Sensibilität. Während das Olfaktorische durch die Aktivitäten der aristokratischen Gesellschaft einen hohen Status erhielt, wurde es über das Mittelalter hinweg in ein streng reglementiertes Tun überführt. So sicher wie das 15. Jahrhundert eine entscheidende Formierungsphase für die Zeremonie bedeutete - dokumentiert durch zahlreiche Nennungen von Duftspielen, Dufttreffen etc. in Tagebüchern und Chroniken - so schwierig ist es, einzelne Orte, Personen oder Jahre als einzelne Entwicklungsschritte zu bestimmen. Unter dem Einfluss des Konzeptes der Wege (michi) bildete sich schließlich die Duft-Kunst $(k \bar{d} d \bar{o})$ heraus. Neben den nicht nur im Westen bekannteren Vertretern der WegeKünste, der Tee- und Blumen-Kunst (chadō bzw. kadō), repräsentiert die DuftKunst einerseits allgemeine Charakteristika des ästhetischen Schaffens der Zeit. Andererseits stellt sie mit ihrem Gegenstand, Duft und Duftmaterialien, sowie den performativen Eigenheiten ihrer Aufführung besondere Anforderungen an die sinnliche und ästhetische Sensibilität. Im Zentrum der Veranstaltungen stehen als Hauptgegenstand Dufthölzer und ihre Düfte, jedoch erscheinen diese nur als ein Teil eines materiell wie auch performativ fest vorgegebenen Rahmens. $\mathrm{Zu}$ diesem gehören verschiedene Utensilien und Werkzeuge, Dekoration und Arrangement von Mobiliar und Raum, Dichtung und Literatur sowie nicht zuletzt die Gemeinschaft der Teilnehmer und die Führung ihrer Körper in klar bestimmten Choreographien. Damit ist das Riechen in dieser spezifischen „olfaktorischen Kultur“ nicht einfach nur eine sinnliche Wahrnehmung, sondern ein Ergebnis einer Vielzahl physischer, motorischer und kognitiver Prozesse. ${ }^{4}$

Eine endgültige Normierung dieser Prozesse erfolgte mit dem Eintritt in die Edo-Zeit (1603-1868), wo schließlich das vorgeschriebene Protokoll, Handlungsmuster und Konventionen in zahlreichen Lehrtraktaten niedergeschrieben wurden. Wie in den übrigen Künsten auch lag die Blüte der Duftzeremonie in der großen Anzahl an Menschen aus aufstrebenden Bürgertum und Händlerschaft begründet, die aus dem Interesse an kultureller Teilhabe heraus eine Unterrichtung in den Künsten suchten. ${ }^{5}$ Neben der Gründung einer Vielzahl von Schulen und der Etablierung eines entsprechenden Lern- und Lehrsystems

2 Vgl. Yamada 2002: 31-32.

3 Vgl. Matsubara 2012: 24-25.

4 Siehe genauer dazu Vogel 2019.

5 Einen detaillierten Blick auf die soziale wie regionale Herkunftsstruktur der Schüler erlaubt das Schülerverzeichnis der Hachiya-Schule (vgl. Nishiyama 1982: 415-423). 
(iemoto seido) kam es zu der Herausgabe und Zirkulation eines umfassenden Schrifttums, welches sich mit Prinzipien und Durchführung der Duft-Kunst auseinandersetzte. Aufgrund der langen Tradition und hohen Komplexität der Veranstaltungen gab es dementsprechend unterschiedliche Typen an Schriften, die sich in der Art und Weise wie auch dem Fokus der Abhandlung unterschieden. $\mathrm{Zu}$ den Texten, welche die ästhetische Auseinandersetzung mit Duft und DuftKunst dokumentieren, gehören zunächst Sitzungsprotokolle. Während das Abfassen von Protokollen einen festen Bestandteil der Sitzungen darstellt, wurden insbesondere die Mitschriften von einigen wenigen berühmten Sitzungen als Beispiele für den Umgang mit Dichtung und Duft überliefert. ${ }^{6}$ Daneben sind es aber vor allem die (geheimen) Lehrtraktate (hi/densho), die eine zentrale Rolle in der Tradierung der Praxis übernehmen. In diesen Schriften wurden Erklärungen zur ornamentalen wie auch performativen Ausgestaltung der Sitzungen gesammelt, wodurch sie eine zentrale Rolle bei Rekonstruktion und Verständnis von Praxis und Diskurs einnehmen. ${ }^{7}$

Während die meisten dieser Traktate als Abschriften unter den Liebhabern und Praktizierenden der Duft-Kunst kursierten und damit nur eine eher kleine Leserschaft erreichten, gelangte im Jahre 1669 (Kanbun 9) mit dem Kōdō hidensho 香道秘伝書 (Die geheimen Lehrschriften zur Duft-Kunst) erstmals eine gedruckte Schrift in Umlauf. Die zahlreichen, in den folgenden fast hundert Jahren erschienenen annotierten Versionen des Textes belegen die weite Zirkulation wie auch dessen hohen normativen Status. Beim Kōdō hidensho handelt es sich jedoch nicht um eine eigens für eine neue Leserschaft verfasste Schrift, sondern vielmehr um eine Kompilation von Abschriften aus dem vorhergegangenen 16. Jahrhundert. Bei den einzelnen Teilen handelt es sich um:

- das Sōshin hikki 宗信筆記 (Notizen Sōshins) von Shino Sōshin 志野宗信 aus dem Jahre 1501 [Kōdō kokoromochi no koto 香道心持の事];

- das Kō awase shiki 香合式 (Zum Zeremoniell des Duftholz-Vergleichs) von Shino Shōha 志野省巴 aus dem Jahre 1558 [Meikō awase no shikata 名香合の しかた];

- das Takakatsu hikki 隆勝筆記 (Notizen Takakatsus) verfasst von Takebe Takakatsu 建部隆勝 aus dem Jahre 1573 [Kō no hikki 香之筆記];

- das Kō no ki 香之記; Autor und Jahr unbekannt, allerdings muss es nach dem Tod Takebe Takakatsu verfasst worden sein [Kō shidai 香次第];

6 Diese Protokolle sind im Wesentlichen die beiden im Samidare nikki 五月雨日記 abgedruckten Treffen Rokuban kō-awase 六番香合 (1479) und Rokushu takimono-awase 六種薰物合 (1478) sowie das Shino Sōshin-ke meikō-awase 志野宗信家名香合 (1501).

7 Eine weitere Variation davon bilden die Dialoglehrschriften. 
- das Setsugetsu kashū 雪月花集 (Eine Sammlung von Schnee, Mond und Kirschblüten) von Shino Sōshin aus dem Jahre 1574;

- das Meikō mokuroku 名香目録 (Verzeichnis berühmter Hölzer) von Shino Sōon 志野宗温 aus dem Jahre 1574 [Rokujū isshu kōmei 六十一種香名];

- das Jitchu kō no ki 十烓香之記 (Aufzeichnung zum Zehner-Abbrenn-Spiel); Autor sowie Jahr unbekannt [Jukumi kō no ki 十組香之記];

- das Kōro no zu 香炉の図 (Illustrationen von Räuchergefäßen) von Kyūōsai Sōnyū 岁翁斎宗入 aus dem Jahr 1573; sowie

- das Sore kō o kiku no meitoku 夫聞香之明徳 (Dies ist die Herrlichkeit Dufthölzern zu lauschen) von Suichikuan Dōsan 翠竹庵道三; aus dem Jahre 1575 [da es sich dabei um einen kurzen Brief von Dōsan an Takakatsu handelt, erscheint der Text in der modernen Forschung auch als Takakatsu ni okuru bun 隆勝に贈る文]. ${ }^{8}$

In ihrer Gesamtheit gebündelt im Kōdō hidensho übten diese einzelnen Texte wesentlichen Einfluss auf die Entwicklung der Duft-Kunst aus und prägten den Diskurs in der Edo-Zeit. Dies soll jedoch nicht darüber hinwegtäuschen, dass es ein wesentliches Problem bei der Bestimmung von Autor- bzw. Herausgeberschaft gibt - sowohl in Bezug auf das Kōdō hidensho wie auch auf die einzelnen zugrundeliegenden Teile. Zunächst zum Kōdō hidensho: Es ist weder möglich, einen Kompilator noch einen Herausgeber der Schrift genau zu bestimmen. Die einzelnen Teile wurden nicht mit einem Vor- oder Nachwort versehen und selbst ein sortierendes Inhaltsverzeichnis ist nicht vorhanden. Diese Tatsache lässt vermuten, dass es sich nicht um einen Connaisseur der Duft-Kunst, sondern eher um einen kommerziell orientierten Herausgeber gehandelt haben muss. Dieser Mangel an einer kompetenten Einführung in die Kunst lässt ebenso die Zuschreibung an Takebe Takakatsu zweifelhaft erscheinen, ${ }^{9}$ da sowohl strukturelle Eigenheiten der Traktate wie auch die deutliche Distanz seiner eigenen Lebensdaten mit der Veröffentlichung ${ }^{10}$ problematisch ist.

8 Es ist wichtig zu betonen, dass alle dieser Schriften ihren Titel erst im Jahre 1739 (Genbun 元文4) mit der Herausgabe einer überarbeiteten Auflage des Kōdō hidensho unter dem Titel Kaisei Kōdō hidensho 改正香道秘伝書 erhielten. Vor diesem Zeitpunkt existierten sie nur als „namenlose“ Fragmente im Kōdō kihan und Kōdō hidensho. Neben der Betitelung wurden die einzelnen Teile auch einer Neuordnung unterzogen und mit Kommentaren versehen (vgl. Midorikawa/Yamane 2005, 204). Für eine bessere Handhabung einerseits und einen leichteren Vergleich mit anderer Forschungsliteratur andererseits werden auch hier diese nachträglich zugewiesenen Titel verwendet; zusätzlich sind in den eckigen Klammern soweit vorhanden weitere alternative Bezeichnungen/Titel für die Traktate angegeben.

9 Diese Meinung vertritt Jinbo 2003: 149.

10 Vgl. Horiguchi 2009: 230-231. 
Im deutlichen Gegensatz zu den Umständen der Veröffentlichung des Kōdō hidensho sind die einzelnen kompilierten Traktate jeweils (zumeist) klar mit Autorschaft und Jahr der Niederschrift versehen. So handelt es sich nominell um Schriften der ersten drei Generationen der Shino-Schule, Shino Sōshin, Shino Sōon und Shino Shōha; weiterhin Takebe Takakatsu, Kyūōsai Sōnyū sowie Suichikuan Dōsan. In Frage steht hier jedoch die Historizität der genannten Personen. Insbesondere Shino Sōshin, der neben Sanjōnishi Sanetaka bis heute oft als einer der Urväter der Duft-Kunst herangezogen wird, ist als historische Figur äußerst problematisch. Ob und wie er tatsächlich gelebt hat, lässt sich nicht eindeutig nachvollziehen; seine führende Rolle in der Entwicklung der Duft-Kunst ist somit wohl ebenso eher fiktiv. ${ }^{11}$ In Bezug dazu schrieb auch schon der edozeitliche Mönch Ningai 忍鎧 (1670-1752), eine berühmte Autorität im Feld der Duft-Kunst, dass es keine direkten Zeugnisse von Sōshin selbst geben würde und alle als solche gekennzeichneten Schriften Fälschungen wären. ${ }^{12}$ Nicht auszuschließen ist die Möglichkeit, dass einzelnen Schriften oder einige ihrer Teile nachträglich verfasst oder Fälschungen sind.

Abseits der problematischen Herausgeber- und Autorschaft stellt das Kōdō hidensho jedoch einen wichtigen Schritt in der Verbreitung der Ideen und Praktiken der Duft-Kunst dar. Das Erscheinen von gedruckten Lehrtraktaten hatte einen wesentlichen Effekt auf die Verbreitung und Organisation von Wissen, dass sich mit der Praxis und Geschichte der Duft-Kunst auseinandersetzt. Es fand bis zu einem gewissen Grad eine Normierung von Wissen statt, welches die (fiktive) Geschichte und Persönlichkeiten der Duft-Kunst wie auch die Performanz der eigentlichen Veranstaltungen und Spielweisen betraf. Manche Facetten der enorm komplexen und vielfältigen Kunst wurden in früheren Traktaten stärker ausgeführt (ideengeschichtliche Prinzipien beispielsweise im Kōdō kihan 香道規範, gegen Ende des 16. Jahrhunderts), manche wiederum in späteren (so zum Beispiel die Duft-Spiele im Kōdō ran no sono 香道蘭之園, im späten 18. Jahrhundert). ${ }^{13}$ Wesentliche Bestandteile finden sich jedoch hier im Kōdō hidensho - zusammengefasst für eine breite Leserschaft. Im Folgenden wurden die ersten beiden der im Kōdō hidensho enthaltenen Traktate, das Sōshin hikki sowie das Kō awase shiki übersetzt.

11 Zur Historizität bzw. Fiktionalität für die Duft-Kunst wichtiger Personen siehe Honma 2014, insbesondere $25-64$ und 70-122.

12 So beispielsweise in Kōdō yodan 香道余談, 231 (enthalten in Midorikawa/Yamane 2008). 13 Soweit nicht anders angegeben, sind die folgenden Traktate, auf die in Fließtext und Fußnoten verwiesen wird, enthalten in Band 10 der vom Geinōshi kenkyūkai herausgegebenen Reihe Nihon shomin bunka shiryō shūsei. Das Kōdō ran no sono wurde herausgegeben von Ozaki und Kunyūsha. 


\section{Kōdō hidensho 香道秘伝書}

$[1]^{14,15}$ Zur Umsicht bei der Duft-Kunst: Wird eine Veranstaltung mit DuftkettenSpiel ${ }^{16}$ abgehalten, wird die dazu geladene Anzahl von Gästen bis zum Vortag eingeladen. [Die Geladenen] bringen dazu eine Anzahl von Dufthölzern mit, die sich in einer gute Verbindung (en 縁) abbrennen ${ }^{17}$ lassen sollten. Dies ähnelt wohl den Verknüpfungen bei der Kettendichtung (renga) u.ä. Wenn beispielsweise [das Holz mit dem Namen] Kumoi 雲井 abgebrannt wird, sollte Ariake 有 明 folgen; nach dem Lauschen von Utatane うたたね ist es gut Nesame ねさめ o.ä. abzubrennen. Bei Dufthölzern [der Kategorie] Jahreszeiten oder auch Liebe ${ }^{18}$ ist es überaus wichtig, dass in einer guten Verbindung abgebrannt wird. Allerdings ist es kaum möglich, immer [für eine gute Verbindung passende] Dufthölzer dabei zu haben. [Insbesondere] wenn viele Dufthölzer herumgegeben werden, ist es schwierig, nur auf deren Verbindung zu achten.

[2] Bei Veranstaltungen aus dem Stehgreif ist es angemessen, die vorhandenen Dufthölzer abzubrennen ohne auf deren Verbindung zu achten. Wenn man [in solch einer Situation dann sogar] eine Verbindung erreicht, gibt es nichts Vergleichbares. Bei ungeplanten Veranstaltungen überlegte Verbindungen zu erreichen, ist überaus schwierig und so ist nichts dagegen einzuwenden, schlicht alle mitgebrachten Dufthölzer abzubrennen.

[3] Bei den Dufthölzern, [die mit ihrem Namen eine Beziehung zu den] Jahreszeiten [haben], kommt - auch wenn man nicht mit der Kunst der Dichtung (kadō 哥道) vertraut ist - die jahreszeitliche Stimmung aus dem Herzen der Dichtung. Den Frühling trägt insbesondere Wakanage 若菜, den Sommer Natsukusa 夏草 und Kakitsubata かきつはた, den Herbst Yaegiku 八重菊 und Momiji もみち und den Winter Hatsuyuki 初雪, Kansō 寒草 und andere als Stimmung in sich. Als Dufthölzer [der Kategorie] Liebe sollte man wissen Tamakura 手枕, Shinobu しのふ oder andere abzubrennen. Da es zu jeder [der Kategorien] viele

14 Grundlage dieser Übersetzung bildet Abdruck und Transkription des Kōdō hidensho in Midorikawa/Yamane 2005.

15 Die Nummerierung der Absätze wurde für eine bessere Übersichtlichkeit im Zuge der Übersetzung hinzugefügt.

16 Takigumi no kō oder auch takitsugi-kō ist ein Duftspiel, bei dem von den Teilnehmern nacheinander Dufthölzer abgebrannt werden, wobei darauf geachtet wird, dass die Namen der jeweiligen Hölzer einen sinnvollen (thematischen) Zusammenhang bilden.

17 An dieser Stelle wird den Formulierungen des Traktates gefolgt. Tatsächlich werden die Hölzer nicht in einer Flamme verbrannt, sondern nur durch Auflegen auf durch heiße Kohlen erhitzte Asche geräuchert.

18 Die Dufthölzer wurden gemäß ihrer Namen in die auch in der Dichtung üblichen Kategorien Jahreszeiten, Liebe, Vermischtes etc. eingeordnet. 
Duftholz-Sorten gibt, sind hier jeweils eine oder zwei zum Überdenken niedergeschrieben.

[4] Nachdem zwei oder drei überaus gute Dufthölzer herumgegangen sind, sollte Tanka 丹霞, Jingai 沈外oder ein ähnliches [Holz] abgebrannt werden; danach ist der Zustand der Sitzung erneuert und ein jeder bereit, [aus seinen mitgebrachten Dufthölzern] mit Bedacht auszuwählen und abzubrennen. Eine solche Umsicht ist das Wichtigste.

[5] Zum Abbrennen von Dufthölzern: Der Gastgeber sollte mit dem Abbrennen beginnen. Jedoch ist nichts dagegen einzuwenden, wenn abhängig von den Bedingungen der Versammlung ein anderer [als erster] abbrennt. Wenn derjenige, der das Feuer [d.h. Kohle] in das Räuchergefäß einfüllt, sich mit den Grußworten „Nun bitte ich, das erste abzubrennen“ an die Versammlung richtet, sollte mit dem Abbrennen begonnen werden. Beim Lauschen ${ }^{19}$ der Dufthölzer wird beim Gast am obersten Platz begonnen und das Räuchergefäß einmal herumgegeben; wenn es wieder vor dem Zeremonienmeister angekommen ist, wird es ein weiteres Mal herumgereicht, sodass man ein jedes Duftholz zwei Mal erhalten kann. Sollten indessen mehr als zehn Leute anwesend sein, wird es beim einmaligen Durchgang belassen.

[6] Beim Lauschen von Dufthölzern sollten Alt wie Jung über eine ihrer Stellung angemessene Haltung Bescheid wissen. Dafür gibt es Gründe. Auch wenn Jugendliche sich gut [in der Welt der] Dufthölzer auskennen, so ist es überaus unnütz, dies zur Schau zu tragen. [Außerdem] ist es in hohem Maße schlecht, wenn man einem Duft lange lauscht. Weiterhin mit einem Ausdruck, dass man den Duft erkannt hat, gleichgültig prätentiös zu lauschen, wirkt unleidenschaftlich und ist schlecht. Von den Älteren in der Runde von den Dufthölzern zu erfahren, nach deren Namen zu

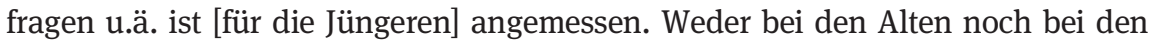
Jungen ist es jedoch angebracht, lange einem Duft zu lauschen.

[7] Beim Lauschen das Räuchergefäß zu sich hin zu neigen und zu lauschen, ist schlecht. Es kommt vor, dass das $\operatorname{Silber}^{20}$ herunterfällt. Oder aber das Duftholz rutscht vom Silber und fällt in die Asche. Ein gewisser Herr handhabte das Räuchergefäß einmal äußerst ungeschickt und ein Stück des berühmten Ranjatai [蘭奢待], ${ }^{21}$ welches die Eigenschaft besitzt, neun weitere Male abgebrannt zu

19 In Anlehnung an buddhistische Konzepte, nach denen die Lehre Buddhas sowohl über die Ohren als auch die Nase aufgenommen werden kann, wird auch in der Duft-Kunst die Geruchswahrnehmung als „Lauschen“ (kiku) bezeichnet. Siehe dazu bspw. das 10. Kapitel des Vimalakīrti-Sutra und die Erklärung im Traktat Kō mondōshū 香問答集, 530.

20 Die Holzstückchen liegen nicht direkt auf der Asche, sondern auf einem kleinen Plättchen aus Silber bzw. Perlmutt.

21 Eines der zehn berühmten und damit wertvollsten Dufthölzer Japans. Die zehn berühmten Sorten (jisshu no meikō) sind: Hōryūji, Tōdaiji, Shōyō 逍遥, Miyoshino みよし野, 
werden, viel herunter. Ohne das es zehn Male abgebrannt wurde, ging es verloren.

[8] Das Räuchergefäß anzuheben, mit wildem Atem zu lauschen und sich den Duft mit der Hand zuzufächeln, ist entsetzlich. Dafür gibt es Gründe.

[9] Wenn das von einem selbst abgerannte Holz die Aufmerksamkeit der Anderen erhält, ist es nicht nötig dem mit bescheidenen Worte trivialisierend zu begegnen. Es ist nämlich ein öffentliches Gut. Wenn ein- oder zweimal deutlich nach dem Namen [des Duftholzes] gefragt wird, sollte er gesagt werden.

[10] Beim Durchreichen des Duftholzes sollte man an seinen Nächsten eine Verbeugung richten. Auf höfliche Weise eine Verbeugung an die gesamte Versammlung zu richten, ist nicht notwendig.

[11] An dem Tag, an dem man zu einem Dufttreffen aufbricht, macht man keinen Raumduft (soradaki). Es ist nicht notwendig $\mathrm{zu}$ sagen warum. Man nimmt auch kein Räucherwerk (takimono) u.ä. in die Hände. Außerdem verwendet man auch keine Duftbeutel.

[12] Auch bei Tee-Treffen, wenn nach dem Tee Dufthölzer hervorgeholt werden, ist an das eben genannte zu denken und auf Raumduft und das Verbrennen [von Dufthölzern] in Feuerbecken und Feuerstelle zu verzichten. Auch [beim Besuch] in einem anderen Haus, selbst wenn nach dem Tee-Treffen kein Raumduft stattfindet, sollte man unter allen Umständen um die Möglichkeit, Duftholz abbrennen zu müssen, wissen und sich mit Sorgfalt um die mitzunehmenden Dufthölzer kümmern.

[13] Beim Lauschen von Dufthölzern ist es falsch, die Asche im Räuchergefäß zu betrachten. ${ }^{22}$ Dafür gibt es Gründe. Nachdem das Duftholz herumgegangen ist, kann man sich [die Asche] ansehen. Wenn Connaisseurs die Asche stoßen, kann man darum bitten und diese ansehen, bevor das Duftholz [herumgegeben wird].

[14] Beim Lauschen von Dufthölzern sollte man sich, auch wenn vornehme Personen [von höherem Rang] an der Versammlung teilnehmen, als Teil der Lauschenden in die Gesellschaft einfügen. Unter keinen Umständen ist [abseits] auf der Veranda zu lauschen.

Hokekyō 法華経, Kōjin 紅塵, Koboku, Yatsuhashi 八橋, Nakagawa 中川 und Rokitsu 盧橘 [Hanatachibana 花橘].

22 In Abhängigkeit von Anlass bzw. Formalitätsgrad der Veranstaltung werden in die Asche in den Räuchergefäßen vor dem Abbrennen der Dufthölzer Muster eingebracht. Während sie ursprünglich in Verbindung zu Yin-Yang und Fünf-Elemente Lehre standen (siehe dafür z.B. das Kōdō kihan 香道規範, 389), wurde ihr Umfang in der Edo-Zeit erheblich reduziert und das Einbringen des Musters damit nur noch zum Marker der Formalität. 
[15] Beim Herumgeben eines Duftholzes sollte in der Runde nicht ungestüm mit einem Fächer hantiert werden. Überhaupt ist bei der Verwendung des Fächers Zurückhaltung zu üben. Dafür gibt es Gründe. Wenn er allerdings aufgrund der Jahreszeit verwendet wird, ist darauf $\mathrm{zu}$ achten, dass dieser langsam und mit Vernunft gehandhabt wird.

[16] Beim Abbrennen eines berühmten Duftholzes (meikō) ein großes Stückchen zu verbrennen, ist ein weit verbreiteter Fehler. Wenn die Versammlung möglicherweise aus vielen Menschen besteht, sollte ein Stückchen in etwas größerer Größe abgebrannt werden. Ist [die Teilnehmerzahl] allerdings nicht so [groß], sollte im Hinblick darauf das Holz auf ein entsprechendes Maß geschnitten werden. Bei fünf bis sechs Teilnehmer [An dieser Stelle bricht der Text des Originals ab.]

[17] Es gibt Leute, die das Silberblättchen ins Feuer werfen und verbrennen, wenn ein Duftholz darauf angebrannt ist. Dies ist ein Fehler. Immer wenn es anschmort, kratzt man es mit einem kleinen Messer ab.

[18] Beim Auflegen und Abnehmen des Silbers wird es mit Fingern und Duftholzstäbchen (kōbashi) an seiner Kante gegriffen; die Duftholzstäbchen werden etwas tiefer als die Finger platziert und [das Silber] in das Räuchergefäß gelegt. Beim Abnehmen verfährt man genauso. Es ist nichts dagegen einzuwenden, nur die Finger ohne die Stäbchen zu verwenden.

[19] Die Maße für das Silber betragen 9 bu [27mm] zum Viereck, wobei die Ecken jeweils $1 \mathrm{bu}$ [3mm] abgeschrägt sind.

[20] Nachdem die Dufthölzer herumgereicht worden sind, wird [das Letzte] unmittelbar ein weiteres Mal auf das Silber gelegt und abgebrannt; vom Gastgeber wird es dann an einer Seite des Zimmers aufgestellt. Zum genauen Ort gibt es eine mündliche Überlieferung.

[21] Beim Abbrennen von Ryūzenkō 竜涎香, Kasugano 春日野 und ähnlichen Räucherwerk-Sorten ist das Silber zu wechseln. Unter keinen Umständen ist mit dem Silber abzubrennen, auf denen berühmte Dufthölzern abgebrannt werden. Ferner werden [diese Sorten] nicht vor und nicht in der Mitte einer Duftholz-Sitzung abgebrannt. Sie sollten am Ende abgebrannt werden. Dies ist selbstverständlich.

[22] Beim Lauschen von Kasugano ist besondere Vorsicht geboten. Der Grund dafür ist, dass es, wenn es fünf oder sechs Personen passiert hat, gewiss und ganz plötzlich verfliegt. Vor langer Zeit hatte sich ein gewisser Herr darauf nicht vorbereitet und so einen Fehler begangen. Um die [richtige] Handhabung muss man wissen.

[23] Wenn sich Leute [das Abbrennen eines weiteren] Duftholzes wünschen, ist es schlecht zu sagen, „Brenn’ noch eins“. Sie sollten mit den Worten „Brenne bitte ein [weiteres] Stückchen ab“ ihren Wunsch äußern. 
[24] Beim Wunsch nach einem [weiteren] Duftholz, ist eines der entsprechenden Jahreszeit angemessen. Allerdings sind bei Connaisseurs, die einen guten Ruf im Lande haben und weithin bewandert sind, Dufthölzer der Jahreszeit, Ga 賀 sowie Uwadaki 上薰 angemessen. Als Higashiyama-dono [Ashikaga Yoshimasa] zur [Feier des] Neubaus des kleinen Versammlungsraumes einlud, erhielt ich die Weisung, eine Sorte zu Beginn des Herumgebens des Räuchergefäßes zu bestimmen und ich brannte Yaegaki 八重垣 ab. Die verschiedensten Dinge liegen dieser Entscheidung zugrunde. Dazu gibt es eine mündliche Überlieferung.

[25] Wenn das Feuer [d.h. die Kohle] in das Räuchergefäß eingefüllt wird, kommt es vor, dass sich die Hitze sehr stark bis zum Boden durchzieht, das Räuchergefäß heiß und kaum zu halten ist. Wenn dies passiert, ist in ein schön anzusehendes Gefäß Wasser einzufüllen und das Räuchergefäß ungefähr drei $b u$ [9mm] [tief hineinzustellen]; wenn der Boden abgekühlt ist, holt man es heraus.

[26] Wird kurz nach dem Umzug in ein neues Haus eine Duft-Versammlung abgehalten, sollte mit einem Enten-Räuchergefäß gelauscht werden. Ist kein Enten-Räuchergefäß bereitgestellt, sollte man mit vorgetäuschter Unwissenheit sagen: „Wenn ein Enten-Räuchergefäß vorhanden ist, so sollte man mit diesem Lauschen“.

[27] Bei einem auf einem Tablett stehenden Räuchergefäß trägt man mit der linken Hand das Tablett, greift mit der rechten das Räuchergefäß, und reicht es so in den Raum. Das Annehmen und Weitergeben [des Räuchergefäßes] während der Sitzung erfolgt ebenso auf dem Tablett stehend.

[28] Menschen von hohem Rang lauschen während das Räuchergefäß auf einem Tablett steht. Bei Kriegerhäusern wird bis hin zum Rang des Daimyō den Dufthölzern gelauscht. Doch obwohl auch diese mit der Hand das Räuchergefäß umfassen, lauschte ein gewisser Herr Izumi Soundso ohne seine Hand anzulegen, was ein überaus abscheulicher Anblick war.

[29] Bei einfachen Leuten wird [das Räuchergefäß] nicht auf dem Tablett stehend angereicht. Es kommt vor, dass das Tablett in der Schmucknische oder aber vor dem Ehrenplatz abgestellt wird. Das Räuchergefäß sollte man [jedoch in jedem Falle] alleine anreichen.

[30] Beim Anreichen des Räuchergefäßes an vornehme Personen wird das Räuchergefäß auf die linke Hand gestellt, die rechte Hand an den Bereich des Boden des Räuchergefäßes gelegt und mit einer überaus ehrfurchtsvollen Haltung angereicht. Bei der Annahme wird mit der rechten Hand das Räuchergefäß von oben [gegriffen] und so als ob es [davon] herunterhängt zu sich genommen.

[31] Beim Weitergeben des Räuchergefäßes an Gleichrangige wird es auf die linke Hand gestellt, die Fingerspitzen der rechten an den Mittelteil der Seite des Räuchergefäßes gelegt und angereicht. Der Annehmende hält seine linke Hand 
unter die Hände des Anreichenden, hebt mit seiner rechten Hand das Räuchergefäß so, dass es nicht herunter fällt und stellt es auf seine linke Hand.

[32] Beim Reichen des Räuchergefäßes an Niedrigrangige, greift man den oberen Teil des Räuchergefäßes mit einer Hand, sodass man es nach unten hängend trägt und gibt es weiter indem man es auf die linke Hand des Empfangenden abstellt.

[33] Reicht man das Räuchergefäß Kindern, jungen Menschen oder Frauen an, trägt man es vor die Person und stellt es auf den Tatami ab; man gibt es nicht in die Hand weiter.

[34] An dem Tag, an dem Dufthölzern gelauscht werden soll, sind beim Arrangement der Räuchergefäße in der Schmucknische die Silber zwischen den Boden des Räuchergefäßes und das Tablett zu legen. Wenn das Duftholzkästchen ( $k \bar{o} g \bar{o}$ 香合) hinzu drapiert wird, gilt es unten die Aloe-Hölzer (jinkō) und darüber die Silber zu stellen.

[35] Beim Arrangement von Duftholzkästchen, Duftbeutelchen (kōbukuro 香 袋) und Räuchergefäß sollten fünf Sorten berühmter Dufthölzer [in das Kästchen] hineingetan werden. Vier Sorten sind nach den eigenen Vorlieben auszuwählen und eine Sorte ist von den Dufthölzern der entsprechenden Jahreszeit hinzuzufügen.

[36] Beim Duftholzkästchen als eines der fünf Schmuckelemente im Seitenregal werden fünf Aloe-Hölzer zugeschnitten und zu einer solchen [Abb.] Größe geschnitten - die Dicke ist dieser anzupassen - und uneingewickelt hineingetan. Wenn Menschen danach fragen, ist zu antworten: „Die Länge der Dufthölzer sollte vier bu [12mm], die Breite drei bu [9mm] und die Dicke ein bu [3mm] betragen“. Es werden nur einfache Dufthölzer hineingetan; berühmte Dufthölzer werden nicht hinzugefügt.

[37] Beim Einfüllen des Feuer [d.h. der Kohle] wird [zunächst] die Asche angewärmt. [Dazu] wird ein Feuer in [das Räuchergefäß] hineingetan und darüber die Asche gegeben; ein weiteres Feuer wird bereitgelegt; wenn sich die Asche ein wenig aufgewärmt hat, [das alte] entnommen und das andere in dessen Öffnung getan; wenn [die Asche] zu einem guten Maß aufgewärmt ist, wird sie über das Feuer gegeben und festgedrückt.

[38] Ausschließlich bei Räuchergefäßen zum Lauschen [von Dufthölzern] stößt man die Asche in fünf Felder (gogō 五合). ${ }^{23}$ Für diese Linien des Genusses gibt es Gründe. Dazu gibt es eine mündliche Überlieferung.

[39] Die Asche sollte etwas über den Rand des Räuchergefäßes gehäuft werden. Ist wenig Asche da, muss sie dementsprechend [anders gehandhabt]

23 Siehe vorherige Fußnote. Die Abgrenzung von fünf Feldern durch das Ziehen von fünf Linien stellt das grundlegendste dieser Muster dar. 
werden. Bei viel Asche muss ebenso dieser Menge [entsprechend vorgegangen] werden.

[40] Wenn das eigene Duftholz abgebrannt wird, sollte nicht lange geprüft werden, ob der Duft deutlich hervorkommt oder noch nicht aufsteigt; erst an die Leute weiterzugeben, wenn er wirklich $\mathrm{zu}$ erkennen ist, sollte unterbleiben. Sobald man glaubt, dass der Duft ein wenig aufsteigt, sollte man [das Räuchergefäß] an die Menschen weiterreichen. Ferner kann es auch ohne Probe herumgegeben werden, wenn vorher ein Erfahrener das Feuer gut eingelegt hat und man den Eindruck hatte, dass auch der zuvor herumgegangene Duft gut aufstieg.

[41] Es kann vorkommen, dass das eigene Duftholz ein starkes Feuer erfordert und [deshalb von ihm kein Duft] aufsteigt. [In solch einem Fall] ist allerdings nicht das Silber hinunterzudrücken. Der Grund ist, dass man nicht weiß, welche Sorte von Duftholz der Nachfolgende abbrennen möchte und [das Herunterdrücken des Silbers dafür ebenso] angemessen wäre. Allerdings sollte die Einsicht vorhanden sein, es hinunterzudrücken, wenn der Letzte in der Runde darum bittet.

[42] Wenn Kinder oder Junge einen Duftholz-Wunsch haben, sollten sie es [nicht selber abbrennen, sondern] noch in das Papier eingewickelt mit der Bitte es abzubrennen an die neben ihnen sitzende Person reichen.

[43] An dem Tag, an dem Duft gelauscht werden soll, wird die Asche beim Aufstellen des Räuchergefäßes in der Tokonoma nicht [in fünf Felder] gestoßen. Sie wird nur angehäuft und glatt gestoßen. Wenn kein Lauschen von Dufthölzern geplant ist und nur ein außergewöhnliches Räuchergefäß drapiert wird, sollte wie bei einer [gewöhnlichen] Dekoration des Versammlungsraums die Asche gestoßen und arrangiert werden.

[44] Als Papier zum Einwickeln von Dufthölzern wird kein Sugihara-Papier verwendet. Der Grund dafür ist, dass Gerüche daran hängen bleiben.

[45] Wenn Räuchergefäß und Duftholzschachtel zuvor auf einem Tisch oder einem Brett [zur Dekoration] arrangiert wurden, werden ausschließlich Räuchergefäß und Duftholzschachtel genommen und das Feuer eingefüllt. Sowohl Tisch als auch Brett werden unverändert in der Tokonoma belassen.

[46] Bei Tabletts von mittlerer Größe, viereckigen Tabletts, langen Tabletts oder oval-geschwungenen Tabletts, wird [das Räuchergefäß] stets mit Tablett genommen und das Feuer eingefügt.

[47] Wird bei Wind oder Regen dem Duft gelauscht, wird mit dem eigenen Rücken dem Wind zugewendet gelauscht.

[48] Kommt es bei einem Tee-Treffen zu einer Veranstaltung mit Dufthölzern, sollte nach dem Anreichen eines äußerst dünnen Tees das Räuchergefäß hervorgeholt werden.

[49] Zur Art und Weise Duftbeutel zu binden, gibt es eine mündliche Überlieferung. 


\section{Ende}

Besonders geheime Überlieferungen:

[50] Wenn man meint, die Hitze im Räuchergefäß sei stark, sollte man Dufthölzer verbrennen, die eine starke Hitze benötigen. Dufthölzer, die ein schwache Hitze benötigen, wie beispielsweise Ōgi 扇, sollten nicht verschwendet werden, wenn die Hitze $\mathrm{zu}$ hoch ist. Zu Hölzern, die eine starke bzw. schwache Hitze erfordern, gibt es eine mündliche Überlieferung.

[51] Wenn man glaubt, dass man den Duft nicht erkennt, schaut man sich die Farbe des Holzes an. Es ist selbstverständlich, dass man sich jene beiden berühmten Hölzer ${ }^{24}$ bis hin $\mathrm{zu}$ ihrer Holzfarbe gut anschaut. Auch bei zwei gleichen Hölzern kommt es in Abhängigkeit von ihrem Ort (kidokoro) ${ }^{25} \mathrm{zu}$ außergewöhnlichen Unterschieden.

[52] Dufthölzer, die niemals in der Mitte einer Sitzung herumgegeben werden dürfen, sind Jingai 沈外 und Shakusendan 赤旃檀. Mit anderen Worten: Diese Dufthölzer werden erbeten, um nach dem Abbrennen vortrefflicher Dufthölzer, deren Kolorit zu erhalten. Wenn solcherlei Dufthölzer [deren Eindruck nicht erhalten werden muss] nicht herumgegangen sind, ist es falsch, Jingai abzubrennen.

[53] Taishi 太子 sollte bis zu drei Mal herumgegeben werden. Öfters wird dies nicht gemacht.

[54] Ranjatai 蘭奢待 ist ein Duftholz, das bis zu zehn Mal herumgegeben werden sollte. Seine Schriftzeichen sind ein Geheimnis. ${ }^{26}$

[55] Koboku 古木 sollte bis zu drei Mal abgebrannt werden. Abgesehen von den drei oben genannten Sorten - und es ist nicht nötig, die Zehn Sorten berühmter Dufthölzer hier zu nennen - ist es nicht richtig, deren übrigen Dufthölzer mehrere Male aufzulegen und wiederholt abzubrennen. Weiterhin zeugt es von großer Unkenntnis, wenn über das Für und Wider des dreimaligen Herumgebens diskutiert wird.

[56] Wenn die Hitze im Räuchergefäß schwach wird, wird mit den Duftholzstäbchen von oben ein Loch [in die Asche] gestochen, über das Loch ein Silber gelegt und das Duftholz abgebrannt. Oder aber man nützt die Schwäche [der Hitze] und steckt ein Duftholz, welches man ein zweites Mal abbrennt aufrecht

24 Gemeint sind an dieser Stelle wahrscheinlich Hōryūi 法隆寺 und Tōdaiji 東大寺, zwei der zehn berühmten Hölzer.

25 Zur Sortierung der Dufthölzer dienten die Kategorien der sechs Länder (rikkoku 六国 auch kidokoro 木所: Kyara, Rakoku, Manaban, Maraka, Sumotara und Sasora) sowie der fünf Aromen (gomi 五味). Obwohl sie bisweilen wirklichen Orten zugeordnet werden, sind die Ländern weniger als eine konkrete Herkunfts-, sondern als Gütebestimmung zu verstehen. Die Aromen - süß, bitter, scharf, sauer, salzig - sind dagegen Versuche, die Charakteristika der Düfte zu beschreiben.

26 Sie enthalten die Schriftzeichen Tōdaiji 東大寺, einen alternativen Namen für dieses Holz. 
in das Loch hinein und lässt es so räuchern. Wenn Hochrangige anwesend sind, sollte man [wieder eine Kohle] mit starker Hitze einlegen, damit man hervorragend lauschen kann.

[57] Wenn das Räuchergefäß von vortrefflicher [Form] ist, kommt von den Gästen der Wunsch an den Hausherren, dass es doch bitte in die Tokonoma gestellt wird. Es sollte wohl auch selbstverständlich sein, falls unter den Gästen ein sehr Erfahrener ist, dass dieser den [anderen in der] Versammlung Erläuterungen gibt und es in die Tokonoma stellt. Man muss sich auf die [jeweilige] Sitzung vorbereiten. Wenn es in die Tokonoma gestellt wird, ist es das Wichtigste, die mündlichen Überlieferungen zu [den unterschiedlichen Arten von] Tabletts zu bedenken.

[58] Bei berühmten Dufthölzern wie auch einfachen Aloe-Hölzern kommt es vor, dass Düfte hängen bleiben; um diese zu lösen, gibt es ein besonderes Geheimnis. Eine mündliche Überlieferung lautet: Bei fünf $r y \bar{o}$ [ca. 188g] Aloe nimmt man ungefähr drei Mengen gemahlene Rinde, übergießt diese mit Wasser, tut die Dufthölzer hinein und lässt sie einen Tag und eine Nacht stehen. Danach wäscht man es noch einige Male mit frischem Wasser, entfernt die weißen [Rückstände] und lässt es in der Morgensonne, solange die Sonne noch nicht stark ist, im Schatten trocknen. Zum [anschließenden] Einwickeln in dünnes Papier gibt es eine mündliche Überlieferung.

[59] Zur Vorbereitung des Feuer-Einlegens im Räuchergefäß gibt es vier mündlichen Überlieferungen. Sie sind ein wichtiges Geheimnis: „Die Vorbereitung am Boden“ [Andrücken der Kohle am Boden des Räuchergefäßes], „Die Vorbereitung schwach oben" [eine Kohle mit schwacher Hitze im oberen Teil platziert], „Die Vorbereitung oben, ein wenig heruntergedrückt“ [Eindrücken der Kohle in die aufgehäufte Asche], „Die Vorbereitung in der Erinnerung des Fingers“ [Eindrücken der Kohle mit dem Finger]. Eine mündliche Überlieferung.

[60] Hōryūji ist ein Duftholz, das von [Shōtoku] Taishi aus Indien eingeführt wurde. Für viele Generationen war es lange Zeit unbekannt. Allerdings ist der Spruch überliefert „Wird die Maus aus dem Mauseloch hervorgelockt, verbreitet sie sich in der ganzen Welt". Weil es in der Schatzkammer des Hōryūji aufbewahrt wurde, trägt es seinen Namen. Weiterhin wird es auch nach dem ehrenwerten Namen [des Shōtoku Taishi] Taishi genannt.

[61] Von Tōdaiji wurde unter der Herrschaft des Shogunats [der Ashikaga] bei dem Besuch eines Herrschers in Nara nur einmal ein Stück von einem sun [ca. $3 \mathrm{~cm}$ ] zum Quadrat als Geschenk herausgeschnitten. Dies macht es zu einem sehr seltenen Holz. Dennoch mag es wohl häufiger sein als Taishi. In der Farbe des Holzes sind drei Farben vorhanden.

[62] Miyoshino 三吉野 ist ein dünner [duftender] Teil von Tō[daiji].

[63] Yatsuhashi 八はし [八橋] ist die Rinde von Tō[daiji]. 
[64] Die Benennung von Koboku ist ein Werk Sasaki Dōyos. Es ist eine interessante Überlieferung. Es gibt eine Überlieferung die heißt: „Es verzaubert mehr als das Zweite“. 27

[65] Es heißt, dass Shōyō im Hokkeji 法華寺 in Nara liegt.

[66] [Vom Duftholz Nakagawa gibt es drei Stücke:] Das Erste: Hakunakagawa はく中川, aus dem Besitz von [Sasaki] Dōyo. Das Zweite: Nakanakagawa 中中川, im Besitz der Asakura 朝倉; es ist das Duftholz, welches durch den nach China übergesetzten Mönch Chūsen 中川 mitgebracht wurde. Das Dritte: Happūnakagawa 八風中川, im Besitz der Ashikaga-Shōgune.

[67] Hokke 法華 ist ein Duftholz, welches aus dem kaiserlichen Palast vom Hokkeji auf Kyūshū erbeten wurde. Deswegen heißt es Hokke. Hokkekyō 法華経 ist ein weiterer Name. Es ist ein Duftholz von vornehmer Abstammung.

[68] Bei Natsukusa 夏草 ist der Duft sanft-sprießend und schwer.

[69] Es heißt, dass Kansō 寒草 der innere [weniger ölige] Teil des Holzes Natsukusa ist.

[70] Hitoyadori 人やりとり hat seinen Namen, weil [sein Duft] lange anhält.

[71] Asaji あさち hat ein dünnes und reifes Herz.

[72] Sanae さなへ hat ein Herz von dem es heißt, es sei schwächer als das von Asaji.

[73] Ōgi あふき wurde so benannt, da sein Duft am Ende dünn ist. Der Wind eines Fächers kommt [auch] nicht bis in alle Enden [eines Raumes].

[74] Utatane うたたね hat einen Duft, der verschlafen hervorkommt. In [chinesischen] Schriftzeichen wird es mit sechs Pinselstrichen geschrieben, nämlich , 一二三“.

[75] Iwakado 岩角 liegt im Eshin-in [恵心院] in Nara.

[76] Shakusendan 赤梅檀 ist ein von mir gelauschtes, überliefertes und herausgegebenes Duftholz. Es muss geheim gehalten werden. Es liegt im Burgfried.

[77] Tanka 丹霞 habe ich ausfindig gemacht; von den Umständen habe ich Sanjōnishi [Sanetaka] erzählt, Rat erhalten und es Tanka genannt.

[78] [Der Name] Futami 二見, so heißt es, entstammt der beidseitigen Duftholzschachtel. Weiterhin heißt es [in einer anderen Erklärung], dass er von der Bucht in Futami entstammt. Es ist eine Duftholzschachtel von Sōgi 宗祇.

[79] Sekinoto 関戸 ist ein Duftholz, welches Ranjatai sehr ähnelt. Man muss in gleicher Weise Kenntnisse besitzen wie bei dem berühmten Duftholz [Ranjatai].

[80] Nachdem Taishi oder Tōdaiji abgebrannt wurde, ist, wie schon oben geschrieben, entweder Tanka oder Jingai abzubrennen. Schon bei zwei- oder

27 Schon in einigen edozeitlichen Kommentaren zum Kōdō hidensho wurden die letzten beiden Sätze dieses Absatzes als in ihrem Sinn unverständlich und irrtümlicherweise hinzugefügt bezeichnet und aufgrund dessen gelöscht. 
dreimaligem ernsten Abbrennen vergeht die Stimmung. So gibt es seit alten Zeiten die Regel, diese zwei Sorten abzubrennen und sich daran zu erfrischen.

[81] Während des Lauschens von Dufthölzern, wenn das Feuer im Räuchergefäß gewechselt wird, sollten nicht die Nase geputzt oder anderweitige Handlungen zum Herrichten der eigenen Erscheinung getätigt werden. Solcherlei Dinge sind stets mit äußerster Umsicht zu unternehmen.

[82] Bei Räuchergefäßen in Form einer Ente ist das Silber in einer Ecke an der Seite der Brust aufzulegen. [Es gibt] eine mündliche Überlieferung.

[83] Zum Weiterreichen eines Räuchergefäßes in Form einer Ente: [die Gefäße] werden unter den Flügeln getrennt; befindet sich der Kopf an der Seite des Rumpfes, wird mit dem Kopf an die eigene linke Seite gedreht gelauscht. Dementsprechend wird es in der Runde immer weitergereicht.

[84] Wird [das Gefäß] am Leib getrennt und der Kopf ist am Flügelteil angebracht, wird die Brust an die eigene rechte Seite gedreht und gelauscht. Beim Weiterreichen wird es in die entgegengesetzte Richtung gewendet und weitergegeben. In einer mündlichen Überlieferung heißt es, dass der Schwanz nicht in die Richtung vornehmer Personen gerichtet werden darf.

[85] Wenn im Räuchergefäß die Hitze sehr heiß ist, gibt es eine Methode, das Holz an die Seite des Silbers zu legen.

[86] Allgemein sind Räuchergefäße in der Form von Lebewesen so wie die in Entenform anzunehmen und weiterzugeben.

[87] Lauscht man aus einem Räuchergefäß mit Trigramm-Orakelzeichen (hakke) muss man wissen, dass das Trigramm der entsprechenden Jahreszeit nach vorne gerichtet werden muss. Die Genusslinien in der Asche sowie das Arrangement auf dem Tablett sind wie oben angeführt. Wenn man in dieser [grundlegenden] Sache beim Lauschen fehlschlägt, ist es sehr merkwürdig, wenn man darauf hinweist, dass es gemäß der Jahreszeit Arten gäbe, Genusslinien in die Asche einzuziehen.

Die obigen Absätze sind mündliche Überlieferungen. Von jungen Jahren an habe ich mich über viele Jahrzehnte mit innigsten Bitten an Sanjōnishi [Sanetaka] [gewandt], [bis] er mir diese anvertraute. Auch wenn es die eigenen Kinder sind, sollte [diese Schrift] verschlossen und als Geheimnis [bewahrt werden]. Es ist überaus wichtig, dass die Lehren nicht an Leute weitergegeben werden, die mit dem Weg nicht vertraut sind. Der Grund dafür: unter den Kennern der chinesischen Dinge (karamono) - Nōami 能阿弥, Shinsō 真相, [Murata] Jukō 村田珠光, Matsumoto [Juhō] 松本珠報 und mir - wurden [die Dinge] gemeinsam in ihrer gesamten Tiefe studiert und untereinander gelehrt; aber es gab gleichzeitig viele, die auch darüber wussten. Die Lehren der Dufthölzer wurden keinem anderen Menschen [außer mir von Sanetaka] weitergegeben; diesem Willen bin ich aufrichtig verbunden und für alle Zeiten in tiefer Dankbarkeit. 
Dies sind die Lehren und so darf dieser Band keinem Außenstehenden gezeigt werden. Bei Menschen, die von einen tiefen Willen und Wissbegierde sind und Entschlossenheit zeigen, sind aus diesen Absätzen einige auszuwählen und zu lehren. Ohne [besondere Bewandtnis in den] Schriftzeichen findet sich oftmals eine Sprache, aufgrund der ich mich geniere und so ist [die Schrift] unter keinen Umständen anderen zu zeigen. Dieser Tage bin ich erschöpft und so habe ich die Sachen wie sie mir eingefallen sind ohne Ordnung niedergeschrieben.

Erstes Jahr Bunki [1501], im 9. Monat [Shino] Sōshin 宗信

[88] Zeremoniell des Vergleichs berühmter Dufthölzer: Von der ersten bis zur zehnten Runde werden jeweils zwei und so insgesamt zwanzig Hölzer herumgegeben. Die Anzahl der Teilnehmer muss auch zehn betragen. So werden pro Person jeweils zwei Sorten Holz ausgegeben. Die Dufthölzer sollte man aus den Zehn [berühmten] Sorten - ausgenommen Taishi und Tōdaiji - oder aus den Fünfzig Sorten ${ }^{28}$ auswählen. Zum Abschluss werden die beiden Sorten [Taishi und Tōdaiji] herumgegebenen. Bei den übrigen Dufthölzern ist das zuerst Abgebrannte das der linken Seite, das zuletzt Abgebrannte das der rechten Seite. Wenn man das linke Duftholz für gut befindet, wird das Spielplättchen [mit der Aufschrift] „Links“ gespielt; glaubt man, dass das rechte Duftholz in seinem Duft das Linke besiegt, spielt man das Spielplättchen „Rechts“. Zur Herstellung der Spielplättchen werden Brettchen aus Zeder mit der Breite 6,5 bu [1,9cm], Länge 1sun 9bu [5,7cm] und weiterhin einer Dicke von $1,5 b u[0,5 \mathrm{~cm}]$ geschnitten und auf deren Oberseite die Namen ${ }^{29}$ geschrieben; auf die Rückseite wird bei einem „Links“ und bei einem „Rechst“ geschrieben. Für die Papiere zum Einwickeln [der Dufthölzer] wird ein dünnes Papier acht Mal gefaltet und an dessen Kante ein schmaler Streifen abgeknickt; der Name des Holzes wird oben geschrieben, der Name des Besitzers [des jeweiligen Holzes] unten notiert; dieses wird vier Mal gefaltet und umgedreht, sodass man [die Schrift] nicht lesen kann. In diese Papiere werden die Dufthölzer eingewickelt. Die Art des Faltens ist wie bei den Spielen, welche die jungen Leute heute als Zehn-Sorten-Duftholzspiel $(\text { jisshu-kō })^{30}$ bezeichnen. $\mathrm{Zu}$ allem gibt es eine mündliche Überlieferung. An der

28 Neben den Zehn berühmten Sorten gab es weitere Listen wie die Fünfzig berühmten Sorten, Einundsechzig berühmte Sorten, Hundertachtzig berühmte Sorten etc.

29 Die Spielplättchen waren nicht mit eigentlichen Namen versehen, sondern mit Symbolen oder Schriftzeichen, sodass sie in der jeweiligen Runde bestimmten Teilnehmern zugeordnet werden konnten.

30 Das Zehn-Sorten-Duftholzspiel kann als die Grundform aller Duftholzspiele gelten. Nach seinem Prinzip sind die meisten Spiele aufgebaut. Anders als der Name nahelegt bilden in dem Spiel nicht zehn, sondern nur vier Sorten von Dufthölzern das Ausgangsmaterial. Von drei Sorten werden jeweils drei Holzstückchen, von der vierten ein Stückchen in jeweils einem 
Veranstaltung, die an jenem Tage in unserem Haus stattfand, ${ }^{31}$ habe ich nicht teilgenommen. So ist sie nicht in meinem Gedächtnis. Generell war in früheren Zeiten kaum von etwas wie „Duftholz-Vergleichen“ (kō-awase) zu hören. Im Hause Sanjōnishi Sanetakas fand jener Räucherwerk-Vergleich (takimono-awase) ${ }^{32}$ statt. Und als zu dem Plan, in Verbindung zu dieser [Sanetakas Veranstaltung] eine Veranstaltung abzuhalten, Zustimmung [von Sanetaka] kam, hatte man das Gefühl, dass sie von großer Besonderheit ist. Sie erhielt Worte des Lobes - dass es eine Veranstaltung sei, die noch in vielen nachfolgenden Generationen berühmt sein würde. An vielen Stellen erhielt sie Unterstützung [von Sanetaka] und konnte durchgeführt werden. Der Richter war Muan 夢庵 [Botanka Shōhaku 牡丹花肖 柏]; um das Nachwort bat man Sanjōnishi Sanetaka. Für die gesamte Versammlung war es ein Ereignis, das niemals aus dem Gedächtnis verschwinden wird.

Was die Abschrift und Weitergabe dieses Bandes betrifft, so wurde er ohne Unterbrechungen von der Zeit [Shino] Sōons 宗温 als Aufzeichnung über die herrschaftlichen Zeremonien überliefert und da nun die Beurteilung von Kuroganegata angewiesen wurde, in dieser Form abgeschrieben. Auch wenn man die Lehren der ersten ungefhr 50 Absätze anderen Menschen zeigt, ist es eine große Unannehmlichkeit, die Schrift in ihrer Gesamtheit anderen Menschen zu zeigen.

Im ersten Jahr Eiroku [1558]

[Shino] Shōha 省巴

\section{Quellen- und Literaturverzeichnis}

Aston, William George (Übers.) (1997): Nihongi: Chronicles of Japan from the Earliest Times to $A D$ 697. Bristol/Tokyo: Ganesha Publishing/Oxford University Press.

gefalteten Papier bereitgelegt. Diese Papiertütchen werden zunächst gemischt und danach in zufälliger Reihenfolge abgebrannt. Es gilt den Duft der Hölzer zu erkennen. Während im Mittelalter bereits unterschiedliche Bezeichnungen für Spiele namentlich nachweisbar sind, aber deren genaue Struktur unklar bleibt, wird heute zwischen Zehn-Sorten-Duftholzspiel bzw. Zehner-Abbrenn-Spiel (jïchū-kō/jutchū-kō 十烓香) unterschieden, wobei beim Zehn-Sorten-Duftholzspiel die drei mehrfach vorhandenen Sorten einmal als Probe gelauscht werden, das Zehner-Abbrenn-Spiel jedoch ohne Probe begonnen wird. Bei ersterem werden die Hölzer bereits in der Proberunde benannt als Holz „Eins“, „Zwei“ oder „Drei“ und als solche schließlich beim Wiedererkennen im Spiel auf dem Antwortzettel vermerkt. Bei letzterem ergibt sich die Benennung beziehungsweise Nummerierung erst im Verlauf des Spiels, wobei das erste Holz und somit auch die erste Antwort stets „Eins“ ist.

31 Gemeint ist das Shino Sōshin-ke meikō awase am 14.6.1501, s.o.

32 Gemeint ist das Rokushu takimono-awase am 10.12.1478, s.o. 
Geinōshi Kenkyūkai 藝能史研究會 (Hrsg.) (1976): Suki: Nihon shomin bunka shiryō shūsei, dai 10 kan 数寄 - 日本庶民文化資料集成 (Materialsammlung zur bürgerlichen Kultur Japans, Bd. 10: Suki). Tokyo: San'ichi shobo.

Honma, Yōko 本間洋子 (2014): Chūsei kōki no kōbunka. Kōdō no reimei 中世後期の香文化. 香 道の黎明 (Duft-Kultur des Spätmittelalters. Morgendämmerung der Duft-Kunst). Kyoto: Shibunkaku shuppan.

Horiguchi, Satoru 堀口悟 (Hrsg.) (2009): Kōdō hidensho shūchū no sekai 香道秘伝書集註の世 界 (Die Welt der Annotierten Geheimen Lehrschriften zur Duft-Kunst). Tokyo: Kasama shoin.

Jinbo, Hiroyuki 神保博行 (2003): Kōdō no rekishi jiten 香道の歴史事典 (Geschichtslexikon zur Duft-Kunst). Tokyo: Hakushobō.

Matsubara, Mutsumi 松原睦 (2012): Kō no bunkashi. Nihon ni okeru jinkō juyō no rekishi 香の文 化史。日本における沈香需要の歴史 (Kulturgeschichte des Dufts. Geschichte des Aloeholzbedarfs in Japan). Tokyo: Yūzankaku.

Midorikawa, Fumiko 翠川文子 / Yamane, Kyō 山根京 (Hrsg.) (2005): Kōdō hidensho. Yonekawa Jōhaku kōdō hidenshō 香道秘伝書・米川常白香道秘伝抄. (Die geheimen Lehrschriften zur Duft-Kunst und Die Auszüge aus den geheimen Lehren zur Duft-Kunst von Yonekawa Jōhaku). Kōsho sōsho, Bd. 1. Tsurugashima: Kōsho ni tanoshimu kai.

Midorikawa, Fumiko 翠川文子 / Yamane, Kyō 山根京 (Hrsg.) (2008): Ningai no kōdō chosaku: Jishukō kurabuyama, Kōdō zoku kurabuyama, Kōe ben'yōroku, Kōdō ben'yōroku, Kōdō yodan, Kūge kōdō ben'yō zakki 忍鎧の香道著作 (Die Schriften zur Duft-Kunst von Ningai). Kōsho sōsho, Bd. 2. Tsurugashima: Kōsho ni tanoshimu kai.

Nishiyama, Matsunosuke 西山松之助 (1982): lemoto no kenkyū 家元の研究 (Studien zum Großmeister[-System]). Tokyo: Yoshikawa kōbunkan.

Ozaki, Saeko 尾崎左永子 / Kunyūsha 薰遊舎 (Hrsg.) (2013): Kōdō ran no sono. Zōho kaiteiban 香道蘭之園. 増補改訂版 (Orchideengarten der Duft-Kunst - ergänzte und revidierte Auflage). Kyoto: Tankōsha.

Vogel, Benedikt (2019): Erleben mit allen Sinnen. Inszenierung von Duft und Ästhetik in den Traktaten zur Duft-Kunst des frühneuzeitlichen Japan. München: ludicium.

Yamada, Kentarō 山田憲太郎 (2002): Kōryō. Nihon no nioi. 香料. 日本のにおい (Duftmaterialien. Der Duft Japans). Mono to ningen no bunkashi, Bd. 27. Tokyo: Hōsei daigaku shuppankyoku. 